\title{
Altered Brain Activation During Visuomotor Integration in Chronic Active Cannabis Users: Relationship to Cortisol Levels
}

\author{
George R. King, Thomas Ernst, Weiran Deng, Andrew Stenger, Rachael M. K. Gonzales, Helenna Nakama, \\ and Linda Chang \\ Department of Medicine, John A. Burns School of Medicine, University of Hawaii, Honolulu, Hawaii 96813
}

\begin{abstract}
Cannabis is the most abused illegal substance in the United States. Alterations in brain function and motor behavior have been reported in chronic cannabis users, but the results have been variable. The current study aimed to determine whether chronic active cannabis use in humans may alter psychomotor function, brain activation, and hypothalamic-pituitary-axis (HPA) function in men and women. Thirty cannabis users ( 16 men, 14 women, 18 - 45 years old) and 30 nondrug user controls ( 16 men, 14 women, 19 - 44 years old) were evaluated with neuropsychological tests designed to assess motor behavior and with fMRI using a 3 Tesla scanner during a visually paced fingersequencing task, cued by a flashing checkerboard (at 2 or $4 \mathrm{~Hz}$ ). Salivary cortisol was measured to assess HPA function. Male, but not female, cannabis users had significantly slower performance on psychomotor speed tests. As a group, cannabis users had greater activation in BA 6 than controls, while controls had greater activation in the visual area BA 17 than cannabis users. Cannabis users also had higher salivary cortisol levels than controls $(p=0.002)$. Chronic active cannabis use is associated with slower and less efficient psychomotor function, especially in male users, as indicated by a shift from regions involved with automated visually guided responses to more executive or attentional control areas. The greater but altered brain activities may be mediated by the higher cortisol levels in the cannabis users, which in turn may lead to less efficient visual-motor function.
\end{abstract}

\section{Introduction}

Cannabis is the most widely used illicit drug in the world. The active ingredient in cannabis is $\Delta$-9-tetrahydrocannabinol (THC), which binds to CB1 receptors located in the CNS. CB1 receptors are most prominent in the basal ganglia, cerebellum, hippocampus, and neocortex (Jager et al., 2006). This distribution of $\mathrm{CB} 1$ receptors suggests that cannabis use may affect motor function, memory, and learning.

Acutely, cannabis impairs psychomotor processing and accuracy (Hunault et al., 2009; Roser et al., 2009), but the effects of chronic cannabis use are more variable (Chang and Chronicle, 2007). Reviews of studies on chronic cannabis users indicated only deficits in learning and memory for new information, but not for other cognitive domains (Fattore and Fratta, 2010; Lundqvist, 2010). However, visuospatial skills and executive function may also show deficits (Fattore and Fratta, 2010; Lundqvist, 2010; Martín-Santos et al., 2010). An understanding of the effects of

Received Aug. 11, 2011; revised 0ct. 21, 2011; accepted 0ct. 24, 2011

Author contributions: T.E., A.S., and L.C. designed research; G.R.K., W.D., R.M.K.G., and H.N. performed research; G.R.K. and R.M.K.G. analyzed data; G.R.K., T.E., A.S., R.M.K.G., and L.C. wrote the paper.

This study was supported by National Institute of Neurological Disorders and Stroke and the National Institute on Drug Abuse (Grants U54-NS/DA-56883, K02-DA020569, 2K24-DA16170), National Center for Research Resources (Grants G12RR003061 and P20RR11091), the Office of National Drug Control Policy, and the Queen's Medical Center. The authors declare no financial conflicts of interest.

Correspondence should be addressed to George R. King, 1356 Lusitana Street, 7 th Floor, Department of Medicine, John A. Burns School of Medicine, University of Hawaii, Honolulu, HI 96813. E-mail: grking@hawaii.edu.

DOI:10.1523/JNEUROSCI.4148-11.2011

Copyright $\odot 2011$ the authors $\quad 0270-6474 / 11 / 3117923-09 \$ 15.00 / 0$ cannabis on visuomotor function has important practical implications. For example, cannabis use may impair driving ability and cognitive motor skills required for driving (Ramaekers et al., 2004; Weinstein et al., 2008).

Gender differences in psychomotor function and cannabis use have been reported. For example, males are less dependent on praxic control than females (Chipman et al., 2002). The praxic system involves the regulation of hand and limb position or movement in the absence of visual or tactile cues. Additionally, females have higher $\mathrm{CB} 1$ receptor protein expression than males do in their blood cells (Onaivi et al., 1999). Lastly, males are more likely to be heavy cannabis users than females (Kohn et al., 2004), while heavy cannabis use predicts subsequent anxiety and depression in females (Patton et al., 2002).

Acute THC can increase cortisol levels (Ranganathan et al., 2009; Taber and Hurley, 2009), while chronic THC administration downregulates CB1 receptors (Romero et al., 1997), similar to the downregulation observed after chronic unpredictable stress or with alcohol (Hill et al., 2005). Stress results in enhanced cortisol levels that may impede visuomotor mapping by selectively impairing the subjects' ability to associate task-relevant stimulus-response pairings while ignoring task-irrelevant stimulus-response pairings (Colzato et al., 2008). Thus, it is possible that THC-induced cortisol release in cannabis users may impair visuomotor function.

To assess the effects of chronic cannabis use and cortisol on visuospatial skills, the current study evaluated chronic, active cannabis users in terms of (1) psychomotor speed, (2) visuomo- 
tor processing using a visually paced finger-sequencing task at two different frequencies during blood oxygen level-dependent (BOLD) fMRI, and (3) possible alterations in hypothalamicpituitary-adrenal (HPA) axis function and cannabis craving in relation to the BOLD signals on the fMRI task.

Based on the literature, we hypothesized that chronic active cannabis users would exhibit slower psychomotor performance, which would correlate with the altered BOLD signals on fMRI. We predicted lesser activation of the primary and supplemental motor areas (Witt et al., 2008) in cannabis users compared with controls. We also predicted that cannabis users would have elevated basal salivary cortisol levels, which would correlate with cannabis craving and BOLD signals.

\section{Materials and Methods}

\section{Subjects}

Sixty subjects, comprising 30 cannabis users (14 females, 16 males) and 30 healthy nondrug users (14 females, 16 males) as controls, were screened and enrolled in the study. The subjects were recruited by fliers, word of mouth, and website advertisements. The current study required two visits. The first visit included a screening evaluation (i.e., medical and drug use histories, physical examination, and determination for study eligibility), saliva sample collection, and the neuropsychological test. The second visit included the fMRI scan (within 1 week of the first visit). The subjects were compensated for their time. Subjects were enrolled only if they fulfilled the following criteria: (1) male or female, aged 18-45 years; (2) residing on the island of Oahu, Hawaii; (3) willing and able to comply with study procedures; (4) able to verbalize understanding of the consent form; and (5) right-handed. Cannabis users had to meet the additional criteria of (1) using cannabis 6-7 d/week for at least one year, and (2) positive urine toxicology test for THC on each day of testing. Exclusion criteria for all subjects included (1) confounding neurological or chronic psychiatric disorder (e.g., multiple sclerosis, stroke, schizophrenia, bipolar disorder), (2) chronic severe medical condition that could confound the analysis of the study (e.g., renal or liver failure, diabetes, chronic hypertension), (3) on medications that could confound the analysis of the study, (4) pregnancy (excluded by urine pregnancy test), and (5) contraindication for MR studies (e.g., ferromagnetic metal implants or severe claustrophobia). Cannabis users were instructed to abstain from smoking cannabis on the day of testing. When they arrived for the study, the subjects were asked when they had last smoked, and they all reported the night before, $\sim 12 \mathrm{~h}$ before the study for all cannabis subjects.

The protocol, flyers, and consent forms were approved by the University of Hawaii Cooperative Institutional Review Board. Following verbal and written consent, all subjects were evaluated with detailed medical and drug use history during face-to-face interviews by trained research staff and by a physician, using structured physical and neurological evaluations. In addition, the subjects were evaluated with the Addiction Severity Index, the Symptom Checklist-90, and the Beck Anxiety and Depression Inventories. To optimize their performance on neuropsychological tests and during functional MRI, subjects were required to test negative on a urine toxicology screen for cocaine, amphetamine, methamphetamine, THC (except for cannabis users), opiates, and benzodiazepenes.

\section{Neuropsychological tests}

During the first session, all subjects completed a neuropsychological test battery sensitive to deficits associated with psychomotor and motor function known to be affected by cannabis use. The battery included measures of: (1) psychomotor speed: Trail Making Test A and Digit Symbol Modalities; (2) fine motor speed: Grooved Pegboard Test, dominant hand and nondominant hand [13]; (3) executive motor control: Trail Making Test B and Letter-Number Sequencing Task; and (4) visuomotor and spatial organization: Rey Osterrieth Complex Figure TestCopy condition. We also assessed the subjects' level of anxiety using the Beck Anxiety Inventory.

\section{Salivary cortisol measurement}

Saliva for cortisol analysis was collected using a Salivette (Sarstedt). Cortisol levels $(\mu \mathrm{g} / \mathrm{dl})$ were assayed using a 96-well plate enzyme immunoassay method (High Sensitivity Salivary Cortisol Enzyme Immunoassay Kit; Salimetrics). Saliva samples for cortisol analysis were collected between 10:30 and 11:30 A.M. for each subject for each visit.

\section{Prescan evaluation and craving measurement}

At the beginning of each study day, subjects filled out a brief questionnaire that evaluated their wellbeing and when they last smoked cannabis. This question was to ensure that they complied with the instructions not to smoke on the study day. Immediately after the brief questionnaire, the cannabis users provided a measure of craving for cannabis, using a 100-point visual analog scale (no craving at all $=0$, the most craving ever experienced $=100$ ).

\section{Imaging procedures}

Image acquisition. All scans were performed on a 3 Tesla Siemens TIM Trio scanner (version VB13; Siemens Medical Solutions), using a 12-channel phase-array head coil. Functional MRI was based on a spiral-in-spiral-out sequence with a specially designed spectral-spatial radio frequency pulse to simultaneously reduce susceptibility signal loss by prewinding the throughplane phase for off-resonance spins and to suppress lipid signal (Börnert et al., 2000; Glover and Law, 2001; Glover and Thomason, 2004; Yip et al., 2009). Images from these two echoes were averaged to improve the signalto-noise ratio and to compensate signal loss from susceptibility throughplane gradient. The pulse sequence parameters were echo time (TE)/ repetition time $(\mathrm{TR})=30 / 2000 \mathrm{~ms}, \mathrm{FOV}=22 \mathrm{~cm}, 28$ slices, $5 \mathrm{~mm}$ thickness, $70^{\circ}$ flip angle, $64 \times 64$ matrix. One hundred twenty-four time points were obtained and the first two volumes were discarded to obtain equilibrium. All subjects also received a structural high-resolution 3D magnetizationprepared rapid gradient echo scan (MP-RAGE; sagittal, TR/TE/inversion time $=2200 / 4.91 / 1000 \mathrm{~ms}, 208 \times 256 \times 144$ resolution) for registration of the functional scans into a stereotactic space and to ensure that no gross structural anomalies were present in these subjects.

Checkerboard task. During the second session, within 1 week of the first session, subjects completed two fMRI scans with different rates of checkerboard presentation. During the task, the subjects were presented with a round checkerboard flickering at a low or high level of difficulty ( 2 or 4 $\mathrm{Hz}$, respectively) via MRI-compatible goggles. Each subject was told to use his or her left hand and to touch the thumb with each of the other fingertips sequentially from the index finger toward the little finger and back again, matching the checkerboard flicker rate (tapping twice per second for the $2 \mathrm{~Hz}$ condition, and tapping four times per second for the $4 \mathrm{~Hz}$ condition). No measures of actual task performance were obtained. The subjects were trained on this task outside of the scanner before they performed the task in the scanner.

\section{Data and statistical analyses}

Behavioral and cortisol data. Data were analyzed using Systat version 10 (SAS Institute). Statistical significance was defined as $p<0.05$ (twotailed). The Mann-Whitney $U$ test was used to compare demographic characteristics of cannabis users and control subjects, since not all of the variables were normally distributed (e.g., nicotine pack years). A twoway ANOVA, with Cannabis status and Gender as the main factors, was used to analyze the neuropsychological data. Significant main effects were analyzed further using Bonferroni's post hoc tests.

fMRI data. Functional MRI data were analyzed using the FMRIB Software Library (FSL 4.1; FMRIB Analysis Group, UK) (Zhang et al., 2009). FSL's Motion Correction using FMRIB Linear Image Registration Tool (MCFLIRT) software was used to correct for head motion. Spatial smoothing was performed with a $6 \mathrm{~mm}$ Gaussian filter. FSL's Brain Extraction Tool (BET) software removed nonbrain tissues. The data were also detrended and high-pass filtered (60 s). Using FLIRT, functional data were first registered to the high-resolution T1-weighted MP-RAGE structural data using a linear full search with 12 degrees-of-freedom (DOF), then the high-resolution data were registered to the Montreal Neurological Institute (MNI) 152 template using a 12 DOF linear full search. The hemodynamic responses were modeled using a doublegamma function. An uncorrected $p$ value threshold of 0.05 was used for the single-subject analysis, which modeled blocks of finger tapping. A 


\begin{tabular}{|c|c|c|c|c|c|c|c|}
\hline & \multicolumn{2}{|l|}{ Controls $(n=30)$} & \multicolumn{2}{|c|}{ Cannabis users $(n=30)$} & \multicolumn{2}{|c|}{$\begin{array}{l}\text { Controls versus } \\
\text { cannabis } \\
\text { (Mann-Whitney } U^{*} \text { ) }\end{array}$} & \multirow{2}{*}{$\begin{array}{l}\text { Cannabis users } \\
\text { (Mann-Whitney } U^{*} \text { ) } \\
\text { Males versus females }\end{array}$} \\
\hline & Males $(n=16)$ & Females $(n=14)$ & Males $(n=16)$ & Females $(n=14)$ & Males & Females & \\
\hline \multicolumn{8}{|l|}{ All subjects } \\
\hline Age (years) & 23 & 24.5 & 21 & 22.5 & 109 & 76 & \\
\hline Education (years) & 14 & 16 & 14 & 14.5 & 114 & 66 & \\
\hline Estimated verbal IQ & 109 & 112 & 102 & 112 & 79 & 91 & \\
\hline Alcohol (days/week) & 1 & 1 & 2 & 2 & 81.0 & 48.0 & \\
\hline Alcohol (years usage) & 4.5 & 8 & 5 & 5 & 158.0 & 69.5 & \\
\hline Alcohol (drinks/episode) & 2 & 3 & 3 & 2 & 94.0 & 73.5 & \\
\hline Alcohol (lifetime exposure, grams) & 2080 & 3840 & 4280 & 5680 & 94.0 & 89.0 & \\
\hline Nicotine (pack years) & 0 & 0 & 0.17 & 0 & 84.0 & 74.0 & \\
\hline \multicolumn{8}{|l|}{ Cannabis use characteristics } \\
\hline Age of first use (years) & & & 14.5 & 16 & & & 65 \\
\hline Frequency of use (days/week) & & & 6.5 & 6.5 & & & 53 \\
\hline Daily use (grams/day) & & & 2 & 1 & & & 89 \\
\hline Duration of use (months) & & & 78 & 63 & & & 100 \\
\hline Lifetime exposure (grams) & & & 4589 & 1962 & & & 95 \\
\hline Craving & & & $20.0 \pm 5.3$ & $50.4 \pm 9.4$ & & & $t_{(28)}=2.9, p=0.007$ \\
\hline
\end{tabular}

*None of the Mann-Whitney group differences were significant.
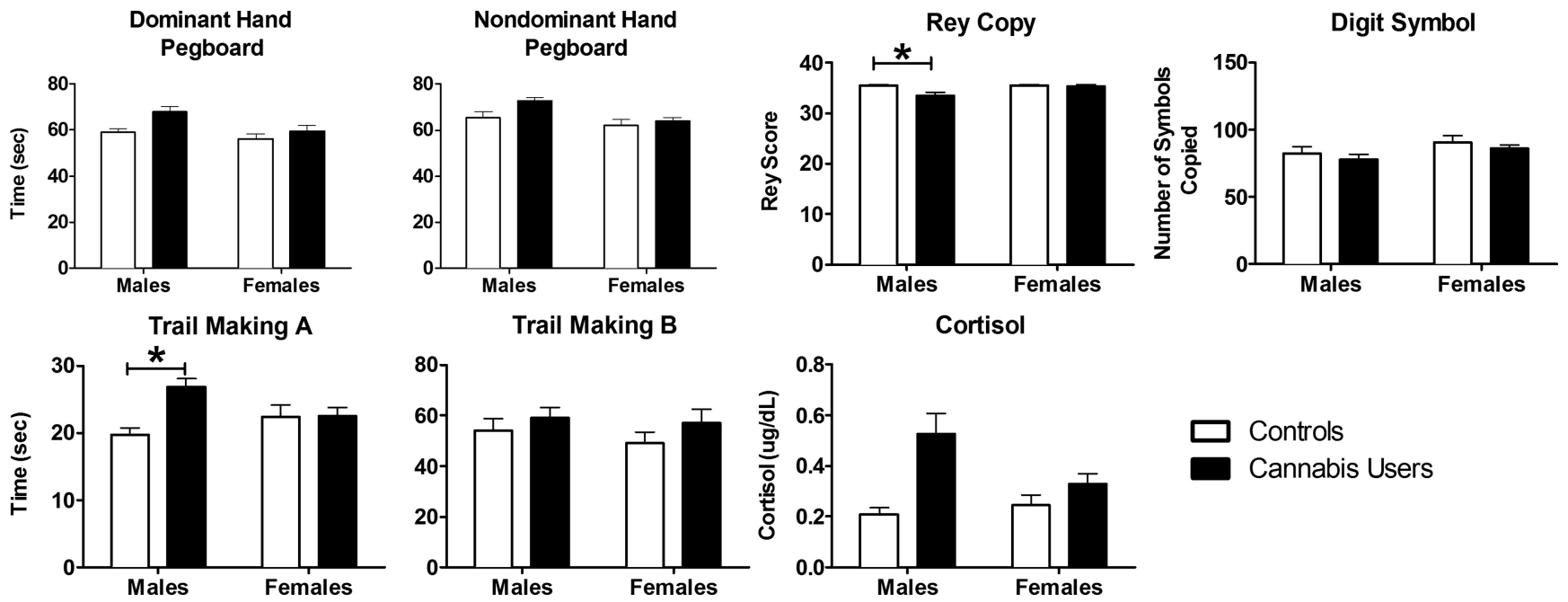

Figure 1. Means and standard errors for the neuropsychological test performance separately for each group and sex. White bars represent the control subjects; black bars represent the cannabis users. There were significant main effects of cannabis use for both Pegboard tasks, the Trail Making A, and Rey Complex Figure (copy condition) tasks. ${ }^{*} p<0.05$, significant difference between the two groups.

two-way mixed-effect ANOVA evaluated the effects of Cannabis status and Gender on BOLD signals, using the general linear model in the FSL FLAME1 procedure. The $Z$ threshold was 2.3 and cluster $p$ thresholds for the group analysis were 0.05 (corrected). Group effects were determined with and without salivary cortisol levels as a covariate to assess the contribution of cortisol to the BOLD signals.

Regions of interest (ROI) analyses were conducted by first masking the average activation map for all subjects for a given flicker frequency by the thresholded zstat map from the Cannabis Status main effect (two-way ANOVA), and for the lingual and superior frontal gyrus (Harvard Cortical Atlas in FSL View). This dual-masked activation map was then thresholded ( $Z=2.3$, fslmaths command $)$ and the resulting activation mask was applied to individual subject data (fsl command featquery) to obtain the BOLD signal strength for each subject and region. The data are expressed as percentage activation of the average activation from all subjects.

\section{Results}

\section{Subject demographics}

Both subject groups were well matched with regards to age, years of education, and estimated verbal IQ (Table 1). Likewise, none
Table 2. Neuropsychological test results, salivary cortisol, and Beck anxiety index

\begin{tabular}{|c|c|c|c|c|c|c|}
\hline \multirow[b]{3}{*}{ Task } & \multicolumn{6}{|c|}{ Main effect (two-way ANOVA) } \\
\hline & \multicolumn{2}{|c|}{ Cannabis use } & \multicolumn{2}{|c|}{ Gender } & \multicolumn{2}{|c|}{ Interaction } \\
\hline & $F_{(1,56)}$ & $p$ & $F_{(1,56)}$ & $p$ & $F_{(1,56)}$ & $p$ \\
\hline Pegboard, dominant hand & 8.86 & 0.004 & 8.14 & 0.006 & 2.03 & 0.16 \\
\hline Pegboard, nondominant hand & 6.39 & 0.01 & 11.89 & 0.001 & 2.92 & 0.09 \\
\hline Trail Making A & 6.57 & 0.01 & 0.26 & 0.61 & 5.78 & 0.02 \\
\hline Trail Making B & 2.31 & 0.13 & 0.81 & 0.37 & 0.01 & 0.91 \\
\hline Rey Complex Figure-Copy & 8.32 & 0.006 & 7.16 & 0.01 & 7.28 & 0.01 \\
\hline Digit symbol substitution & 0.95 & 0.33 & 3.33 & 0.07 & 0.02 & 0.89 \\
\hline Salivary cortisol levels & 8.83 & 0.002 & 1.62 & 0.21 & 1.62 & 0.21 \\
\hline Beck Anxiety Index & 2.40 & 0.13 & 0.72 & 0.40 & 1.90 & 0.17 \\
\hline
\end{tabular}

of the cannabis usage variables were different between male and female users (Age of first use, Amount used, and Lifetime exposure). Alcohol consumption (lifetime grams), duration, frequency of use, and amount per use were not different between the groups, nor were the pack years of nicotine use. Lastly, a physi- 


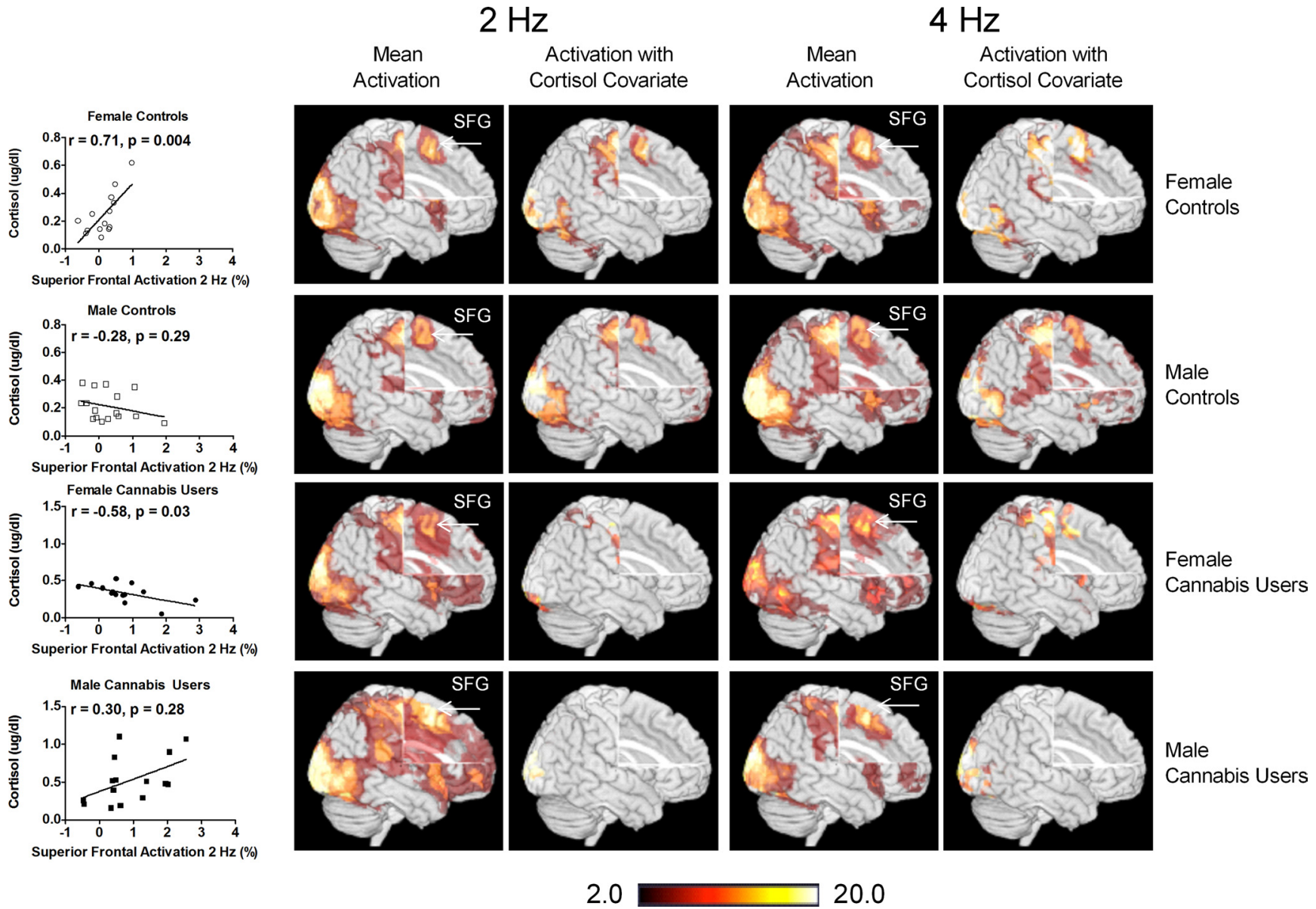

Figure 2. Activation patterns for each group for the 2 and $4 \mathrm{~Hz}$ flicker frequency conditions of the visually paced finger-sequencing task (with and without cortisol as a covariate). Also presented are the correlations between superior frontal gyrus (SFG) activation and cortisol levels for all subjects. fMRI data processing was performed using FEAT (FMRI Expert Analysis Tool) Version 5.98, part of FSL (www.fmrib.ox.ac.uk/fsl).Z (Gaussianised T/F) statistic images were thresholded using clusters determined by $Z>2.0$ and a (corrected) cluster significance threshold of $p=0.05$. The $z$ maps show clusters of statistical significance ( $p<0.05$, corrected), as determined from the FSL analysis.

cian evaluated all except one of the 30 cannabis users and found that only four male cannabis users met criteria for cannabis dependence according to DSM IV.

\section{Psychomotor function}

Cannabis users performed poorer than control subjects on the two Pegboard tasks, the Trail Making A, and Rey Complex Figure Copy task (Fig. 1; Table 2). Compared with females, males performed slower on both Pegboard tasks, but similarly on the Trail Making Task. Lastly, male cannabis users performed the Trail Making A Task $\left(t_{(58)}=3.79, p<0.001\right)$ significantly slower, and the Rey Complex Figure Copy $\left(t_{(58)}=3.32, p<0.01\right)$ tasks significantly less accurately than the male controls. No sexdifferences on the Beck Anxiety Inventory were found between the subject groups (Table 2).

\section{Cortisol and cannabis craving}

Cannabis users had higher salivary cortisol levels than control subjects (Fig. 1; Table 2). In addition, female cannabis users reported significantly more craving than male cannabis users (female craving: $50.4 \pm 9.4$; male craving: $20.0 \pm 5.3 ; t_{(28)}=2.9$, $p=0.007$; Table 1). Cortisol levels did not correlate with craving scores. Neither cortisol levels nor cannabis craving correlated with any measure of cannabis use history or with any measure of psychomotor test performance, neither as a group nor separately for male and female cannabis users.
fMRI during the Finger-sequencing task: $2 \mathrm{~Hz}$ flicker frequency condition

All groups showed activation of brain areas involved in visually guided motor behavior, such as BA 4 (primary motor), BA 17 (primary visual cortex), BA 18 (secondary visual association cortex), and BA 19 (tertiary visual association cortex) (Fig. 2). The cannabis users demonstrated lesser activation in BA 17 and 18 (lingual gyrus and cuneus), but greater activation in BA 6 (superior frontal gyrus) compared with control subjects (independent of gender) (Table 3; Fig. 3). Furthermore, independent of cannabis status, women activated more of the right BA 17 (cuneus) than men (Table 3), while men activated more in the left BA 17 and 18 (lingual gyrus and cuneus) than women.

Lastly, significant group-by-gender interactions were observed on the two-way ANOVA (Table 4; Fig. 4). Compared with male controls, male cannabis users showed significantly less activation of bilateral precentral gyrus (BA 4, 6) and left visual cortex (BA 17, 18). The male cannabis users also had greater activation of right superior frontal gyrus (BA 6) and left superior temporal gyrus (BA 38) in the $2 \mathrm{~Hz}$ condition than male controls. In contrast, female cannabis users activated less of right fusiform gyrus (BA 18) and right middle temporal gyrus (BA 37) compared with female controls. Female cannabis users did not activate more than female controls in any brain region on the $2 \mathrm{~Hz}$ condition. 
Table 3. Significant main effects of drug use and gender from the checkerboard task

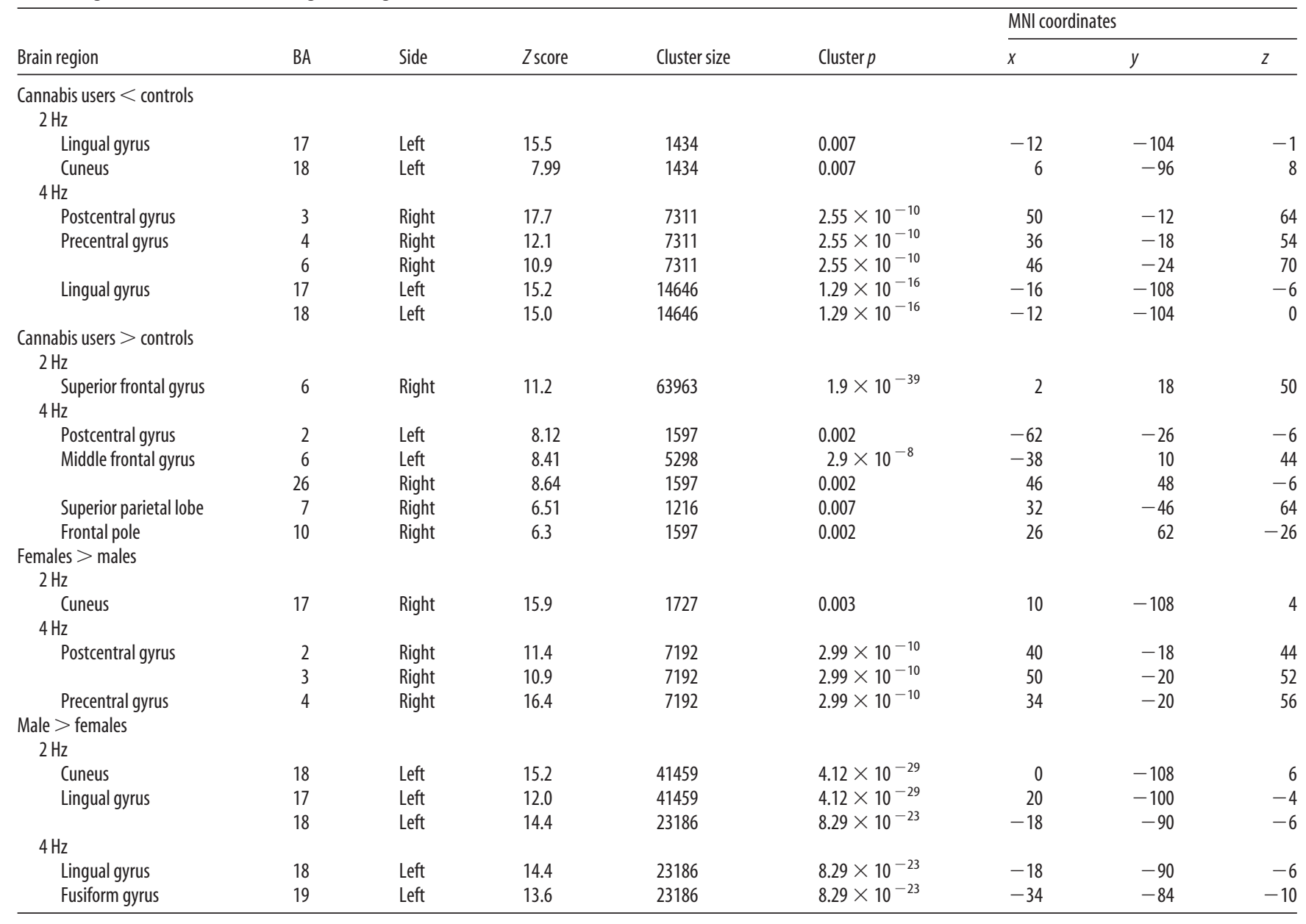

fMRI during the Finger-sequencing task: $4 \mathrm{~Hz}$ flicker frequency condition

On the $4 \mathrm{~Hz}$ condition, cannabis users demonstrated less activation of right postcentral gyrus (BA 3), right precentral gyrus (BA 4,6 ), and left lingual gyrus (BA 17, 18) than controls (Table 3; Fig. 3 ). Conversely, compared with controls, cannabis users showed greater activation of the left postcentral gyrus (BA 2), bilateral middle frontal gyrus (BA 6, 26), right superior parietal gyrus (BA 7 ), and right frontal pole (BA 10) (Table 3 and Fig. 3). Independent of cannabis use, females demonstrated greater activation in right postcentral gyrus (BA 2,3) and right precentral gyrus (BA 4) than males, whereas males demonstrated greater activation of the left lingual gyrus and cuneus (BA 18, 19) than females (Table 3).

Significant group-by-gender interactions were also observed (Table 4; Fig. 4), with male cannabis users showing less activation of left postcentral gyrus (BA 3) and right precentral gyrus (BA 6) than male controls. However, male cannabis users showed greater activation of left superior frontal gyrus (BA 9) and left inferior parietal lobe (BA 40) compared with male controls. In contrast, compared with female controls, female cannabis users demonstrated less activation of right postcentral gyrus (BA 3), left precentral gyrus (BA 6), and right fusiform gyrus (BA 18), but greater activation of bilateral middle frontal gyrus (BA 10, 44, 26) and right superior temporal gyrus (BA 38) compared with female controls.

\section{Cortisol and finger sequencing}

To evaluate the possible role of cortisol in the finger-sequencing task, cortisol levels were entered as a covariate in the averaged analysis for each group (Fig. 2). Due to the covariation of cortisol with the BOLD signals in the superior frontal gyrus, activation was eliminated in all areas except for the occipital lobe in the male cannabis users, and significantly reduced in the visual and motor control areas in the females. In contrast, since both male and female control subjects had lower cortisol levels, their brain activation were only minimally reduced when cortisol was entered as a covariate.

\section{ROI analyses}

Based on the results from the finger-sequencing task, ROIs in the lingual gyrus (visual area) and the superior frontal gyrus (executive control area) were selected for further analyses (Fig. 3). Onebetween (Group), one-within (Flicker frequency) ANOVAs were performed on each ROI. Percentage activation in the superior frontal gyrus was positively correlated with cortisol levels in female controls, but negatively correlated with cortisol in the female cannabis users (Fig. 2). However, activation of the superior frontal gyrus did not correlate with cortisol levels for either the male controls or male cannabis users. Cannabis users had less activation in the lingual gyrus $\left(F_{(1,116)}=13.0, p=0.0004\right)$ and greater activation in the superior frontal gyrus $\left(F_{(1,116)}=11.1, p=0.001\right)$ than the controls. Activation in either the superior frontal or lingual gyrus was not significantly correlated with drug use history or neuropsychological test performance for any group.

\section{Discussion}

The main findings of our study are as follows: (1) active chronic cannabis users had slower performance in psychomotor speed 


\section{$2 \mathrm{~Hz}$}

Cannabis Users < Controls
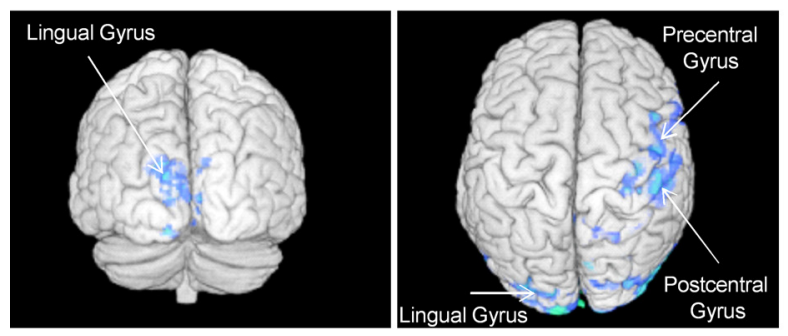

2.0
$4 \mathrm{~Hz}$

Gyrus

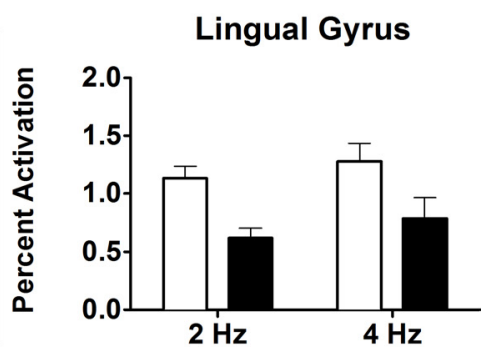

Cannabis Users $>$ Controls
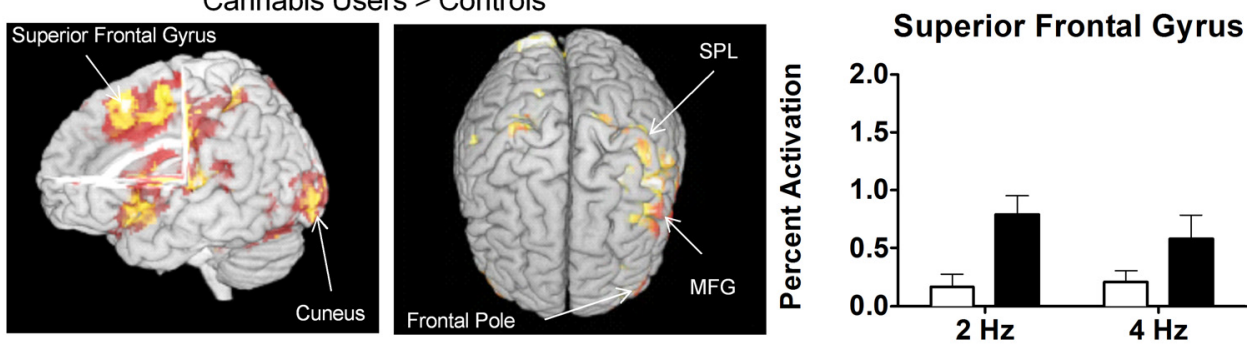

2.0

10.0

Figure 3. Differences in activation between cannabis users and control subjects for the 2 and $4 \mathrm{~Hz}$ flicker frequency conditions of the visually paced finger-sequencing task. Also presented is the percentage activation in the lingual and superior frontal gyri for each group for the 2 and $4 \mathrm{~Hz}$ condition of the visually paced finger-sequencing task. fMRI data processing was performed using FEAT Version 5.98, part of FSL. Z (Gaussianised T/F) statistic images were thresholded using clusters determined by $Z>2.0$ and a (corrected) cluster significance threshold of $p=0.05$. The $z$ maps show clusters of statistical significance ( $p<0.05$, corrected) as determined from the FSL analysis. MFG, Middle frontal gyrus; SPL, superior parietal lobule.

Table 4. Significant cannabis use-by-gender interaction from the checkerboard task

\begin{tabular}{|c|c|c|c|c|c|c|c|c|}
\hline \multirow[b]{2}{*}{ Brain Region } & \multirow[b]{2}{*}{ BA } & \multirow[b]{2}{*}{ Side } & \multirow[b]{2}{*}{ Zscore } & \multirow[b]{2}{*}{ Cluster size } & \multirow[b]{2}{*}{ Cluster $p$} & \multicolumn{3}{|c|}{ MNI coordinates } \\
\hline & & & & & & $x$ & $y$ & $z$ \\
\hline \multicolumn{9}{|c|}{ Male cannabis users $<$ male controls } \\
\hline \multicolumn{9}{|l|}{$2 \mathrm{~Hz}$} \\
\hline \multirow[t]{3}{*}{ Precentral gyrus } & 4 & Right & 11.3 & 4103 & $3.93 \times 10^{-6}$ & 36 & -16 & 50 \\
\hline & 6 & Right & 4.14 & 4103 & $3.93 \times 10^{-6}$ & 32 & -6 & 60 \\
\hline & & Left & 11.2 & 2295 & 0.0001 & -60 & 0 & 34 \\
\hline Lingual & 17 & Left & 14.7 & 4103 & $3.93 \times 10^{-6}$ & -12 & -104 & -10 \\
\hline Cuneus & 18 & Left & 11.1 & 4103 & $3.93 \times 10^{-6}$ & -12 & -108 & 4 \\
\hline \multicolumn{9}{|l|}{$4 \mathrm{~Hz}$} \\
\hline Postcentral gyrus & 3 & Left & 7.34 & 2295 & 0.0001 & -60 & -14 & 30 \\
\hline Precentral gyrus & 6 & Right & 14.8 & 33790 & $1.99 \times 10^{-29}$ & 36 & -18 & 54 \\
\hline \multicolumn{9}{|c|}{ Male cannabis users $>$ male controls } \\
\hline \multicolumn{9}{|l|}{$2 \mathrm{~Hz}$} \\
\hline Superior frontal gyrus & 6 & Right & 14.3 & 45139 & $1.92 \times 10^{-31}$ & 2 & 18 & 50 \\
\hline Superior temporal gyrus & 38 & Left & 12.6 & 45139 & $1.92 \times 10^{-31}$ & -48 & 14 & -12 \\
\hline \multicolumn{9}{|l|}{$4 \mathrm{~Hz}$} \\
\hline Superior frontal gyrus & 9 & Left & 8.56 & 4647 & $1.19 \times 10^{-7}$ & 2 & 22 & 54 \\
\hline Inferior parietal lobe & 40 & Left & 8.41 & 4647 & $1.19 \times 10^{-7}$ & -54 & -32 & 64 \\
\hline \multicolumn{9}{|c|}{ Female cannabis users $<$ female control } \\
\hline \multicolumn{9}{|l|}{$2 \mathrm{~Hz}$} \\
\hline Fusiform & 18 & Right & 8.45 & 946 & 0.04 & 22 & -98 & -14 \\
\hline Middle temporal lobe & 37 & Right & 5.79 & 946 & 0.04 & 56 & -66 & 8 \\
\hline \multicolumn{9}{|l|}{$4 \mathrm{~Hz}$} \\
\hline Postcentral gyrus & 3 & Right & 16.6 & 11133 & $7.63 \times 10^{-14}$ & 50 & -14 & 66 \\
\hline Precentral gyrus & 6 & Left & 10.2 & 11133 & $7.63 \times 10^{-14}$ & -48 & 4 & 62 \\
\hline Fusiform & 18 & Right & 10.9 & 11133 & $7.63 \times 10^{-14}$ & 26 & -94 & -14 \\
\hline \multicolumn{9}{|c|}{ Female cannabis users $>$ female controls } \\
\hline \multicolumn{9}{|l|}{$4 \mathrm{~Hz}$} \\
\hline \multirow{3}{*}{ Middle frontal gyrus } & 10 & Left & 11.5 & 14990 & $5.82 \times 10^{-17}$ & -58 & 16 & 4 \\
\hline & 44 & Right & 8.9 & 2778 & $2.9 \times 10^{-5}$ & 48 & 52 & -2 \\
\hline & 46 & Left & 5.79 & 1115 & 0.01 & -36 & 44 & 12 \\
\hline Superior temporal gyrus & 38 & Right & 7.71 & 14990 & $5.82 \times 10^{-17}$ & 52 & 20 & -24 \\
\hline
\end{tabular}




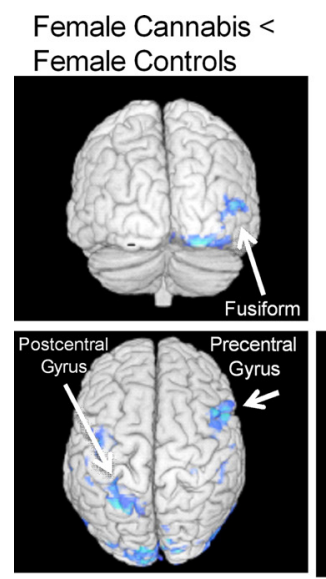

2.0

10.02 .0

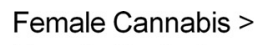

Female Controls

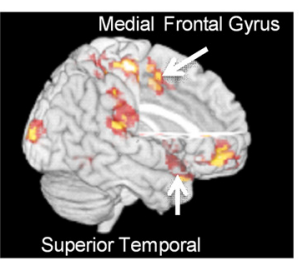

10.0

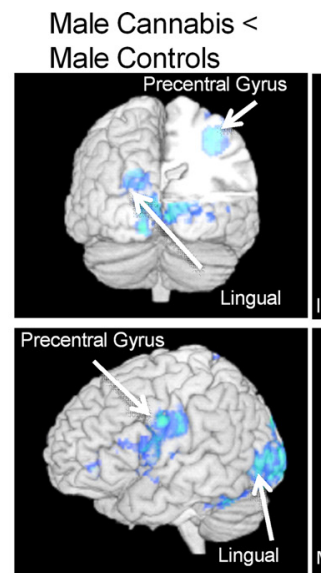

2.0

10.0

Male Cannabis >

Male Controls

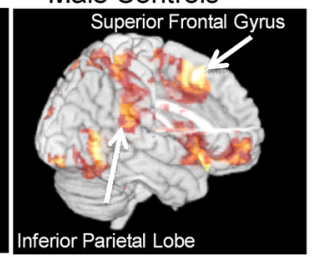

$2 \mathrm{~Hz}$

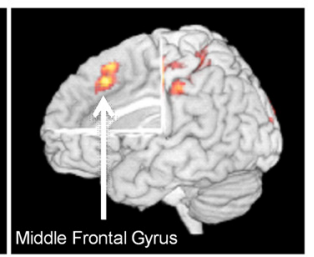

$4 \mathrm{~Hz}$

Figure 4. Differences in activation between female cannabis users and control subjects, and male cannabis users and control subjects, for the 2 and $4 \mathrm{~Hz}$ flicker frequency conditions of the visually paced finger-sequencing task. FMRI data processing was performed using FEAT Version 5.98, part of FSL.Z (Gaussianised T/F) statistic images were thresholded using clusters determined by $Z>2.0$ and a (corrected) cluster significance threshold of $p=0.05$. The $z$ maps show clusters of statistical significance $(p<0.05$, corrected) as determined from the FSL analysis. Cool (blue) colors indicate areas where cannabis users had significantly less activation than controls; hot (red/orange) colors indicate areas where cannabis users had significantly greater activation than controls.

tasks than nondrug users; (2) cannabis users demonstrated lesser activation in the lingual gyrus, but greater activation of the superior frontal gyrus compared with control subjects; and (3) cannabis users, particularly male users, had higher cortisol levels, which may have contributed to their poorer performance and brain activation.

\section{Neurocognitive test performance}

The slower performance on psychomotor tasks in our male cannabis users is consistent with prior findings of decreased visual processing speed (Fried et al., 2005) and impaired visuospatial skills (Bolla et al., 2002) in active chronic cannabis users. The less efficient visuomotor function is not likely related to cannabis craving, as female cannabis users reported more craving, but performed better, than male users. Chronic cannabis use might impair visuomotor performance in the males, but may have a cumulative effect on executive motor controls by attenuating frontal activation in the females.

\section{Finger sequencing task during fMRI}

Primary and nonprimary motor areas control movement (Picard and Strick, 1996), although other areas are also involved when pacing stimuli are used. Visually paced finger sequencing tasks are associated with activation of the bilateral insula, inferior frontal gyrus, occipital lobe, and posterior cerebellum (Zhang et al., 2003), whereas auditory-paced tasks activate BA 44 (Thaler et al., 1995; Witt et al., 2008). Our results are consistent with these findings, as all groups activated the occipital lobe and cerebellum. Lastly, BA 9, 18, and 37 have been implicated in the processing of the visual pacing stimuli (Schmahmann et al., 1999; Vaillancourt et al., 2006), and our subjects activated BA 18 and 37.

\section{Cannabis use and finger sequencing}

Our cannabis users demonstrated less activation of the lingual gyrus (BA 17) than the controls for both flicker frequencies. This area is responsible for visual attention (Smith et al., 2006; Silver et al., 2007). In contrast, the cannabis users demonstrated significantly greater activation of the superior frontal gyrus and brain regions involved in attention (prefrontal and parietal regions, and insula). These areas are also involved in motor planning (Bischoff-Grethe et al., 2004). These differential activation pat- terns between the two groups suggests that the cannabis users shifted from more automated visually guided responses to more executive or attention control regions of the brain.

Our results are consistent with prior findings of lower activation of BA 6 during a self-paced finger-sequencing task in abstinent cannabis users compared with nonusers (Pillay et al., 2004). Our active cannabis users demonstrated decreased activation of the precentral region of BA 6 , while the subjects from the previous report had lower activation in the superior frontal gyrus (Pillay et al., 2004). These discrepancies might be due to differences in the recency of cannabis use or the different levels of task difficulty. The previous study involved a self-paced finger tapping at 1 $\mathrm{Hz}$, which may be more difficult and require more control and attention than the visually paced finger sequencing tasks (Witt et al., 2008) used in our study.

\section{Gender differences and finger sequencing}

We found multiple gender differences, regardless of cannabis use, in activation patterns during the finger-sequencing task. Specifically, men exhibited activation primarily in visuospatial areas, whereas women showed activation primarily in motor planning areas. Our finding of men primarily using visual spatial processing to perform the finger-sequencing task is consistent with a prior study that found males do not use the praxic control system as extensively as females for motor tasks (Chipman et al., 2002).

Furthermore, during the checkerboard tasks, our female subjects showed greater left hemisphere activation than the men, while the males showed greater right hemisphere activation than the females. These findings are consistent with prior fMRI studies of working memory tasks, in which men lateralized their activation to the right, but women activated primarily the left prefrontal and parietal areas (Speck et al., 2000; Bell et al., 2006). The gender-specific lateralization of the hemispheric activation may be related to the working memory that was required for the finger sequencing portion of the task.

\section{Cannabis use by gender interactions and finger sequencing}

Male cannabis users consistently activated the superior frontal gyrus more than male controls, while female cannabis users activated the middle frontal gyrus more than female controls. The superior frontal gyrus is involved in behavioral planning (Meister 
et al., 2004; Del-Ben et al., 2005; Kübler et al., 2006), while the middle frontal gyrus is involved in behavioral inhibition (Bernal and Altman, 2009). Thus, the male cannabis users may have required more effort in the planning of the finger-sequencing task than male controls, while female cannabis users may have had greater difficulty in inhibiting their finger sequencing than female controls (Chen et al., 1995; Thaler et al., 1995; Dagher et al., 1999). The greater effort in planning in the male cannabis users would also account for their slower performance in psychomotor speed tests.

\section{Effects of THC on cortisol}

Since acute administration of THC can increase cortisol levels in humans (Ranganathan et al., 2009), the enhanced cortisol levels in our cannabis users may be due to residual THC in the users' system. Visuomotor mappings of arbitrary stimuli (a round checkerboard) to motor sequences (finger-sequencing) is mediated by a network consisting of the premotor and prefrontal cortices that is impaired by chronic cannabis, but not cocaine, use (Colzato and Hommel, 2008; Colzato et al., 2008). Furthermore, stress and enhanced cortisol levels also weaken visuomotor mappings by selectively impairing the subjects' ability to create task relevant stimulus-response pairings (Colzato et al., 2008). Thus, the elevated cortisol levels may have affected cannabis users' performance by altering brain activation. However, we did not find a correlation between cortisol and the amount of marijuana use, which may be due to tolerance that had developed in these chronic users. As we discussed in the introduction, chronic THC administration downregulates CB1 receptors, and chronic cannabis users typically showed blunted cortisol reactivity (Ranganathan et al., 2009). Since our subjects were all long-term chronic active cannabis users, the relationship between the amount of marijuana used and cortisol no longer exists, either as a group or separated by gender.

\section{Interaction of drug use, gender, and cortisol}

Overall, the current findings suggest interactions between gender and cannabis use on visuomotor integration and planning. First, male, but not female, active cannabis users performed the psychomotor tasks slower than their respective controls. Second, the superior frontal gyrus had greater activation in male cannabis users compared with male controls for both conditions. Since BA 6 has been linked to the executive control for simple motor movements (Sadato et al., 1997), as well as motor initiation and planning (Chen et al., 1995; Thaler et al., 1995; Dagher et al., 1999), the greater activation of BA 6 in male cannabis users suggests less efficient visuomotor motor planning in these subjects.

This hypothesis is consistent with prior research that suggested that visually paced finger sequencing tasks are not automatic and may require sustained attention and effortful learning (Witt et al., 2008). Also consistent with this hypothesis is the finding that abstinent cannabis users showed an altered visualattention network, assessed with parametrically increasing attentional load (Chang et al., 2006).

Less efficient visuomotor integration may be related to greater glucocorticoid exposure in the prefrontal cortex. Prolonged glucocorticoid exposure impairs prefrontal cortical function, resulting in disturbed inhibitory regulation of the HPA axis and behavior (McEwen, 2004). For example, glucocorticoids enhance proinflammatory cytokines in hippocampal cell cultures (MacPherson et al., 2005) and enhance ischemia-induced hippocampal cell loss (Sapolsky and Pulsinelli, 1985; MacPherson et al., 2005). Of note, male cannabis users had the poorest performance in the visuomotor tasks compared with controls, and also had the highest levels of basal salivary cortisol. Furthermore, activation in the motor areas of the male cannabis users covaried with cortisol levels, indicating greater neural requirements with higher cortisol levels.

\section{Limitations of the current study}

The current study has three limitations. First, the accuracy of the self-reported marijuana usage data may be limited. We did perform urine toxicology on these subjects to verify the recent usage of cannabis, but more detailed cannabis usage history with timeline-follow back interviews (Chang et al., 2006) and hair analyses also may be useful. Since subject performance was not monitored during the visually paced finger-sequencing task, it is not known how accurately the subjects performed the task. However, both groups showed activations in the premotor and supplementary motor areas and sensorimotor cortices, which is consistent with performance of visually paced finger-sequencing tasks (for review, see Witt et al., 2008). Lastly, while we posit that the results are mediated by elevated cortisol and prefrontal cortical function, it is possible that the cannabis users were more stressed, and therefore had to be more vigilant, and/or were bored by or less focused on the tasks, which could account for the altered pattern of activation compared with the controls.

In conclusion, chronic active cannabis use is associated with slower and less efficient psychomotor function and visuomotor processing, especially in the male users, which may be related to the cortisol levels.

\section{References}

Bell EC, Willson MC, Wilman AH, Dave S, Silverstone PH (2006) Males and females differ in brain activation during cognitive tasks. Neuroimage 30:529-538.

Bernal B, Altman N (2009) Neural networks of motor and cognitive inhibition are dissociated between brain hemispheres: an fMRI study. Int J Neurosci 119:1848-1880.

Bischoff-Grethe A, Goedert KM, Willingham DT, Grafton ST (2004) Neural substrates of response-based sequence learning using fMRI. J Cogn Neurosci 16:127-138.

Bolla KI, Brown K, Eldreth D, Tate K, Cadet JL (2002) Dose-related neurocognitive effects of marijuana use. Neurology 59:1337-1343.

Börnert P, Aldefeld B, Eggers H (2000) Reversed spiral MR imaging. Magn Reson Med 44:479-484.

Chang L, Chronicle EP (2007) Functional imaging studies in cannabis users. Neuroscientist 13:422-432.

Chang L, Yakupov R, Cloak C, Ernst T (2006) Marijuana use is associated with a reorganized visual-attention network and cerebellar hypoactivation. Brain 129:1096-1112.

Chen YC, Thaler D, Nixon PD, Stern CE, Passingham RE (1995) The functions of the medial premotor cortex. II. The timing and selection of learned movements. Exp Brain Res 102:461-473.

Chipman K, Hampson E, Kimura D (2002) A sex difference in reliance on vision during manual sequencing tasks. Neuropsychologia 40:910-916.

Colzato LS, Hommel B (2008) Cannabis, cocaine, and visuomotor integration: evidence for a role of dopamine D1 receptors in binding perception and action. Neuropsychologia 46:1570-1575.

Colzato LS, Kool W, Hommel B (2008) Stress modulation of visuomotor binding. Neuropsychologia 46:1542-1548.

Dagher A, Owen AM, Boecker H, Brooks DJ (1999) Mapping the network for planning: a correlational PET activation study with the Tower of London task. Brain 122:1973-1987.

Del-Ben CM, Deakin JF, McKie S, Delvai NA, Williams SR, Elliott R, Dolan M, Anderson IM (2005) The effect of citalopram pretreatment on neuronal responses to neuropsychological tasks in normal volunteers: an FMRI study. Neuropsychopharmacology 30:1724-1734.

Fattore L, Fratta W (2010) How important are sex differences in cannabinoid action? Br J Pharmacol 160:544-548.

Fried PA, Watkinson B, Gray R (2005) Neurocognitive consequences of 
marihuana: a comparison with pre-drug performance. Neurotoxicol Teratol 27:231-239.

Glover GH, Law CS (2001) Spiral-in/out BOLD fMRI for increased SNR and reduced susceptibility artifacts. Magn Reson Med 46:515-522.

Glover GH, Thomason ME (2004) Improved combination of spiral-in/out images for BOLD fMRI. Magn Reson Med 51:863-868.

Hill MN, Patel S, Carrier EJ, Rademacher DJ, Ormerod BK, Hillard CJ, Gorzalka BB (2005) Downregulation of endocannabinoid signaling in the hippocampus following chronic unpredictable stress. Neuropsychopharmacology 30:508-515.

Hunault CC, Mensinga TT, Böcker KB, Schipper CM, Kruidenier M, Leenders ME, de Vries I, Meulenbelt J (2009) Cognitive and psychomotor effects in males after smoking a combination of tobacco and cannabis containing up to 69 mg delta-9-tetrahydrocannabinol (THC). Psychopharmacology 204:85-94.

Jager G, Kahn RS, Van Den Brink W, Van Ree JM, Ramsey NF (2006) Longterm effects of frequent cannabis use on working memory and attention: an fMRI study. Psychopharmacology (Berl) 185:358-368.

Kohn L, Kittel F, Piette D (2004) Peer, family integration and other determinants of cannabis use among teenagers. Int J Adolesc Med Health $16: 359-370$

Kübler A, Dixon V, Garavan H (2006) Automaticity and reestablishment of executive control-an fMRI study. J Cogn Neurosci 18:1331-1342.

Lundqvist T (2010) Imaging cognitive deficits in drug abuse. Curr Top Behav Neurosci 3:247-275.

MacPherson A, Dinkel K, Sapolsky R (2005) Glucocorticoids worsen excitotoxin-induced expression of pro-inflammatory cytokines in hippocampal cultures. Exp Neurol 194:376-383.

Martín-Santos R, Fagundo AB, Crippa JA, Atakan Z, Bhattacharyya S, Allen P, Fusar-Poli P, Borgwardt S, Seal M, Busatto GF, McGuire P (2010) Neuroimaging in cannabis use: a systematic review of the literature. Psychol Med 40:383-398.

McEwen BS (2004) Protection and damage from acute and chronic stress: allostasis and allostatic overload and relevance to the pathophysiology of psychiatric disorders. Ann N Y Acad Sci 1032:1-7.

Meister IG, Krings T, Foltys H, Boroojerdi B, Müller M, Töpper R, Thron A (2004) Playing piano in the mind: an fMRI study on music imagery and performance in pianists. Brain Res Cogn Brain Res 19:219-228.

Onaivi ES, Chaudhuri G, Abaci AS, Parker M, Manier DH, Martin PR, Hubbard JR (1999) Expression of cannabinoid receptors and their gene transcripts in human blood cells. Prog Neuropsychopharmacol Biol Psychiatry 23:1063-1077.

Patton GC, Coffey C, Carlin JB, Degenhardt L, Lynskey M, Hall W (2002) Cannabis use and mental health in young people: cohort study. BMJ 325:1195-1198.

Picard N, Strick PL (1996) Motor areas of the medial wall: a review of their location and functional activation. Cereb Cortex 6:342-353.

Pillay SS, Rogowska J, Kanayama G, Jon DI, Gruber S, Simpson N, Cherayil M, Pope HG, Yurgelun-Todd DA (2004) Neurophysiology of motor function following cannabis discontinuation in chronic cannabis smokers: an fMRI study. Drug Alcohol Depend 76:261-271.

Ramaekers JG, Berghaus G, van Laar M, Drummer OH (2004) Dose related risk of motor vehicle crashes after cannabis use. Drug Alcohol Depend 73:109-119.

Ranganathan M, Braley G, Pittman B, Cooper T, Perry E, Krystal J, D’Souza
DC (2009) The effects of cannabinoids on serum cortisol and prolactin in humans. Psychopharmacology (Berl) 203:737-744.

Romero J, Garcia-Palomero E, Castro JG, Garcia-Gil L, Ramos JA, FernandezRuiz JJ (1997) Effects of chronic exposure to delta9-tetrahydrocannabinol on cannabinoid receptor binding and mRNA levels in several rat brain regions. Brain Res Mol Brain Res 46:100-108.

Roser P, Gallinat J, Weinberg G, Juckel G, Gorynia I, Stadelmann AM (2009) Psychomotor performance in relation to acute oral administration of Delta9-tetrahydrocannabinol and standardized cannabis extract in healthy human subjects. Eur Arch Psychiatry Clin Neurosci 259:284-292.

Sadato N, Yonekura Y, Waki A, Yamada H, Ishii Y (1997) Role of the supplementary motor area and the right premotor cortex in the coordination of bimanual finger movements. J Neurosci 17:9667-9674.

Sapolsky RM, Pulsinelli WA (1985) Glucocorticoids potentiate ischemic injury to neurons: therapeutic implications. Science 229:1397-1400.

Schmahmann JD, Doyon J, McDonald D, Holmes C, Lavoie K, Hurwitz AS, Kabani N, Toga A, Evans A, Petrides M (1999) Three-dimensional MRI atlas of the human cerebellum in proportional stereotaxic space. Neuroimage 10:233-260.

Silver MA, Ress D, Heeger DJ (2007) Neural correlates of sustained spatial attention in human early visual cortex. J Neurophysiol 97:229237.

Smith AT, Cotillon-Williams NM, Williams AL (2006) Attentional modulation in the human visual cortex: the time-course of the BOLD response and its implications. Neuroimage 29:328-334.

Speck O, Ernst T, Braun J, Koch C, Miller E, Chang L (2000) Gender differences in the functional organization of the brain for working memory. Neuroreport 11:2581-2585.

Taber KH, Hurley RA (2009) Endocannabinoids: stress, anxiety, and fear. J Neuropsychiatry Clin Neurosci 21:109-113.

Thaler D, Chen YC, Nixon PD, Stern CE, Passingham RE (1995) The functions of the medial premotor cortex. I. Simple learned movements. Exp Brain Res 102:445-460.

Vaillancourt DE, Mayka MA, Corcos DM (2006) Intermittent visuomotor processing in the human cerebellum, parietal cortex, and premotor cortex. J Neurophysiol 95:922-931.

Weinstein A, Brickner O, Lerman H, Greemland M, Bloch M, Lester H, Chisin R, Sarne Y, Mechoulam R, Bar-Hamburger R, Freedman N, EvenSapir E (2008) A study investigating the acute dose-response effects of $13 \mathrm{mg}$ and $17 \mathrm{mg}$ delta 9-tetrahydrocannabinol on cognitive-motor skills, subjective and autonomic measures in regular users of marijuana. J Psychopharmacol 22:441-451.

Witt ST, Laird AR, Meyerand ME (2008) Functional neuroimaging correlates of finger-tapping task variations: an ALE meta-analysis. Neuroimage 42:343-356.

Yip CY, Yoon D, Olafsson V, Lee S, Grissom WA, Fessler JA, Noll DC (2009) Spectral-spatial pulse design for through-plane phase precompensatory slice selection in $\mathrm{T} 2{ }^{*}$-weighted functional MRI. Magn Reson Med 61:1137-1147.

Zhang JX, Leung HC, Johnson MK (2003) Frontal activations associated with accessing and evaluating information in working memory: an fMRI study. Neuroimage 20:1531-1539.

Zhang J, Anderson JR, Liang L, Pulapura SK, Gatewood L, Rottenberg DA, Strother SC (2009) Evaluation and optimization of fMRI single-subject processing pipelines with NPAIRS and second-level CVA. Magn Reson Imaging 27:264-278. 